\title{
Politique
}

\section{Démocratie et libéralisme : pour une approche historico-théorique}

\section{Guy Laforest}

Numéro 13, printemps 1988

Crise de décision

URI : https://id.erudit.org/iderudit/040585ar

DOI : https://doi.org/10.7202/040585ar

Aller au sommaire du numéro

Éditeur(s)

Société québécoise de science politique

ISSN

0711-608X (imprimé)

1918-6584 (numérique)

Découvrir la revue

Citer cet article

Laforest, G. (1988). Démocratie et libéralisme : pour une approche

historico-théorique. Politique, (13), 87-109. https://doi.org/10.7202/040585ar d'utilisation que vous pouvez consulter en ligne.

https://apropos.erudit.org/fr/usagers/politique-dutilisation/ 


\title{
DÉMOCRATIE ET LIBÉRALISME: POUR UNE APPROCHE HISTORICO-THÉORIQUE
}

\author{
Guy Laforest \\ Université de Calgary
}

Peut-on écarter l'approche historique lorsqu'on essaie d'appréhender des idéologies, des concepts aussi fondamentaux dans la tradition de la pensée occidentale que ceux de libéralisme et démocratie? C'est le pari qu'a tenté de relever Stéphane Dion dans un texte récemment publié par cette revue ${ }^{1}$. Oeuvrant à l'enseigne de la mesure et de la lucidité, Dion a proposé une synthèse sur la place de la démocratie libérale dans le monde contemporain. Réflexion lucide, car l'auteur est beaucoup plus conscient que d'autres néo-libéraux de l'oscillation du libéralisme entre les valeurs de la démocratie, l'égalité substantielle et la liberté réelle. Plaidoyer mesuré, puisque le libéralisme garde une importante flexibilité lorsqu'il est confronté avec ce que l'auteur appelle les quatre légitimités démocratiques ${ }^{2}$.

1. Stéphane Dion, «Libéralisme et démocratie: plaidoyer pour l'idéologie dominante», Politique, (9), 1986, pp. 5-38. Stéphane Dion et moi avons eu une discussion publique au sujet de son article lors du congrès de la Société québécoise de science politique, ACFAS, Ottawa, 20 mai 1987. Le texte qui suit est une version remaniée de celui que j'avais présenté à cette occasion. Je voudrais remercier les évaluateurs de cette revue pour leurs suggestions et leurs commentaires.

2. Ibid., pp. 8 ss.

Politique, 13 (Printemps 1988). 
Écrivant dans une atmosphère idéologique favorable à un individualisme plus musclé, à un libéralisme plus agressif, l'auteur avance des thèses qui paraîtront justes à une foule de lecteurs. Nous songeons en particulier, dans le contexte du Québec postréférendaire, à la conception du rapport entre individu et communauté qui transpire dans sa réflexion. Un des objectifs du présent article c'est d'empêcher l'adhésion, selon nous imprudence et insuffisamment justifiée, à une vision ultra-individualiste du libéralisme. Il est par ailleurs trop facile de faire de la renonciation à l'histoire un postulat méthodologique. Cela mène à simplifier grandement les sens possibles du libéralisme et de la démocratie pour nos sociétés. Chez Dion, l'oubli de l'histoire permet de mésestimer la contingence d'une philosophie de l'homme et de la société sous-jacente à la définition libérale de la liberté, celle de l'individualisme atomiste. En prenant conscience des paramètres historiques de cette philosophie, nous pourrons envisager ici des façons autres de concevoir la théorie et la pratique de la liberté. Fermé à la dimension historique, Dion va jusquà faire de la démocratie directe une invention de l'esprit, n'ayant pas plus existé «à Athènes qu'en Suisse ou ailleurs ${ }^{3}$. Sur la force d'un tel rejet, l'auteur peut rejeter l'idée d'une certaine pertinence de la démocratie directe à l'époque contemporaine, comme il peut aussi réserver une place congrue au thème de la participation. À notre avis, ces phénomènes méritent davantage d'attention au cœur de notre actualité politique.

Le nécessaire raccordement entre théorie politique et histoire est le pilier sur lequel repose tout notre propos. Nous en montrerons d'abord la pertinence dans les débats contemporains en pensée politique. Par la suite, nous examinerons d'une manière critique les fondements de l'individualisme atomiste. La conception de la

3. Ibid., p. 8 . 
liberté qui en découle devrait en perdre son auréole d'évidence. Nous rappellerons enfin, grâce à l'histoire des idées politiques, la trajectoire de la démocratie antique et la récupération de certains de ses idéaux à l'ère moderne par l'humanisme républicain.

$S$ 'imposent à ce moment-ci quelques remarques préliminaires. Notre approche décevra sûrement le lecteur qui voudrait savoir, une fois pour toutes, ce qu'il faut entendre par libéralisme et démocratie. Il ne s'agit pas de remplacer des certitudes par d'autres. Que la signification des concepts en question n'est pas épuisée par les analyses qu'en propose Dion, c'est tout ce que nous voulons démontrer pour l'instant. Afin de réaliser cet objectif très circonscrit, nous ferons appel au panthéon d'une tradition de pensée connue sous le nom d'humanisme civique ou républicain. Les libéraux donnent parfois l'impression ces temps-ci qu'ils sont les seuls penseurs dotés de légitimité dans la modernité occidentale. Le tableau que nous tracerons de l'humanisme républicain, qui ira forcément au plus pressé, souhaite aussi invalider une telle croyance ${ }^{4}$. Replacer l'histoire au cœur même du projet de la théorie politique, employer la critique pour faire vaciller une infrastructure conceptuelle, cela est bien loin de constituer toute la tâche de l'élaboration d'une pensée politique indépendante. Il n'est pas dit que nous y parviendrons ${ }^{5}$. Le présent article indique que cela nous amènerait à prendre davantage en considération les obligations qui découlent de l'appartenance à une communauté politique, à tenir compte aussi de la situation pratique d'une telle communauté. Les libéraux font comme s'il n'y avait pas de limites au-delà desquelles la défense du quant-à-soi virerait à l'absurde.

4. On ne prétend pas en quelques paragraphes résumer la pensée des Machiavel, Montesquieu, Rousseau. On peut toutefois indiquer, à grands traits, ce que les partisans de l'humanisme civique croient trouver dans leurs travaux.

5. Les historiens de la pensée politique ne font pas nécessairement les théoriciens les plus originaux. 


\section{Théorie politique et histoire}

Dans le cadre d'un colloque sur le renouvellement de la pensée politique, John Dunn proposa quelques orientations susceptibles de rapprocher la théorie politique de la réalisation de son objectif fondamental, à savoir contribuer à la formulation du diagnostic de notre situation pratique, éthico-politique, et à l'identification des moyens nous permettant de faire face à une telle situation ${ }^{6}$. Pour atteindre un tel objectif, la théorie politique devrait nous aider à saisir le comment et le pourquoi des dimensions économique, politique et sociale de nos vies; elle devrait également nous permettre de voir comment l'on pourrait établir d'une façon cohérente la plausibilité de manières autres d'envisager le réel.

Dunn est assez représentatif de ce que nous croyons être les tendances dominantes de la théorie politique contemporaine. L'heure nous semble être à une certaine modestie dans les prétentions du théoricien. L'intellectuel universel, pour employer le langage de Foucault, le monarque de la pensée capable d'appréhender l'idée de la justice et d'instaurer le régime idéal, ou à tout le moins apte à saisir le contour des vérités morales, est en retraite ${ }^{7}$. Sartre pas plus que Platon ne conviennent dans une conjoncture caractérisée sinon par le nihilisme, du moins par un certain pessimisme civilisationnel. Moins optimiste, forcée au réalisme par le piteux état de notre monde et par les dangers qui le menacent, la théorie politique, plus spécifiquement dans le monde anglo-saxon, peut toujours se rabattre sur une certitude: celle de son existence. Ce ne fut pas toujours le cas.

6. John Dunn, «Reconceiving the Content and Character of Modern Political Community", communication présentée dans le cadre d'un colloque sur le renouvellement de la pensée politique, tenu à l'université d'Ottawa, octobre 1986, p. 1.

7. Michel Foucault, Power/Knowledge, New-York: Pantheon Books, 1980, p. 128. 
Si certains libéraux d'aujourd'hui prétendent pouvoir se passer de l'histoire, leurs prédécesseurs d'il y a trente ans multipliaient les oraisons funèbres pour la théorie et la philosophie politiques ${ }^{8}$. Les problèmes du langage et de la-logique de la science monopolisaient l'attention de la philosophie analytique. La science politique positiviste ouvrait en mimétisme avec les méthodes des sciences de la nature. Elle rêvait de constructions axiomatiques, érigées dans l'environnement aseptisé de la neutralité axiologique. La théorie politique traditionnelle, avec ses quêtes hésitantes, son entreprise entachée par un lourd bagage normatif, paraissait définitivement mise à l'écart. Dans les paramètres néo-évolutionnistes de la pensée anglo-américaine d'alors, la modernité occidentale était affublée du statut de zénith, de summum s'imposant à toute l'humanitée. À l'époque de la fin des idéologies, la théorie politique était devenue quasiment inutile pour ceux qui considéraient la démocratie libérale à l'américaine comme la manifestation politique d'un tel zénith. Dans ce contexte qui était aussi celui de la guerre froide, il fallait déplacer l'effort théorique du côté du Tiers-Monde; les théories du développement et de la modernisation sont issues de telles préoccupations.

Elles sont nombreuses les raisons qui, allant s'accélérant à partir des années soixante, ont mené à une ébullition, à un retour en force de la théorie politique ${ }^{10}$. Des carrefours importants sur

8. Richard Bernstein, The Restructuring of Social and Political Theory, Pennsylvania: University of Pennsylvania Press, 1978, p. 57.

9. Voir à ce sujet l'article de Carl Pletsch, "The Three Worlds, or the Division of Labor, circa 1950-1975", Comparative Studies in Society and History, XIII(4), 1981, pp. 565-590.

10. Dans l'ordre de la pensée, sans prétention à l'exhaustivité, il faudrait mentionner le minage des certitudes de la tradition analytique par le conventionnalisme de Wittgenstein à propos du langage, l'analyse des mutations paradigmatiques et des processus des révolutions scientifiques chez Thomas Kuhn, puis aussi l'érosion, provoquée par des critiques telles celles de Charles Taylor, des prétentions de la science et de la théorie politique positivistes à la neutralité axiologique. Dans les horizons plus larges de la société, la guerre du Vietnam, la crise de légitimité de l'ordre socio-politique et économique constitutif de 
cette voie, on en trouve dans les travaux de Rawls et Nozick, qui ont tous deux relevé en dépit de leurs divergences réciproques le niveau théorique de la discussion à l'intérieur de la problématique des droits subjectifs ${ }^{11}$. Toujours dans le monde anglo-saxon, une nouvelle réceptivité a permis de mieux accueillir les contributions de la pensée continentale, de l'herméneutique à la phénoménologie, de l'École de Francfort aux divers aspects du nietzschéisme français ${ }^{12}$. Une autre dimension intrinsèque à cette évolution, qui nous mène au cœur de notre propos, c'est la participation de l'histoire au redémarrage de la théorie politique.

Autour des travaux de John Dunn et de Quentin Skinner, une nouvelle école a vu le jour en histoire des idées politiques, porteuse d'importantes conséquences pour la théorie politique dans son ensemble. L'école de Cambridge, si nous pouvons nous permettre cette expression, part d'une insatisfaction au sujet de la façon de concevoir le dialogue et les rapports entre les théoriciens et théories politiques d'hier et d'aujourd'hui. Elle milite en faveur d'une étude des idées politiques qui prend vraiment au sérieux leur dimension historique, de façon à ne pas tomber dans le piège de l'anachronisme, de la fausse universalité ${ }^{13}$. Il n'y a pas une

l'ère post-industrielle, ont également contribué à ébranler les certitudes de ceux qui avaient placé la théorie politique sur une voie d'évitement. La même chose vaut pour les écueils rencontrés par les modèles assez simplistes des théoriciens du développement et de la modernisation.

11. John Rawls, A Theory of Justice, Oxford: Oxford University Press, 1972. Voir aussi Robert Nozick, Anarchy, State and Utopia, New-York: Basic Books, 1974, pour une exposition du capitalisme libertaire à partir d'une théorie des droits.

12. Parmi les ouvrages qui témoignent bien de cette réceptivité, voir Fred Dallmayr et Thomas McCarthy, Understanding and Social Enquiry, Notre-Dame: University of Notre-Dame Press, 1977.

13. Quentin Skinner, The Foundations of Modern Political Thought, Cambridge: Cambridge University Press, 1978, vol. 1, pp. XI-XIII. Voir aussi John Dunn, "The Identity of the History of Ideas", dans John Dunn, Political Obligation in its Historical Context, Cambridge: Cambridge University Press, 1980, p. 20. Sur la question du recouvrement des intentions, voir John Dunn, The Political Thought of John Locke. Cambridge: Cambridge University Press, 1969, p. 9. 
seule grande tradition dans la pensée occidentale, un vocabulaire demeuré immuable de Platon jusqu'à nous; il faut donc faire preuve de sensibilité face aux mutations linguistiques des arsenaux de la pensée politique. Pour comprendre les discours politiques d'auteurs tels Machiavel ou Locke, pour saisir leurs intentions, il faut reconstituer les univers idéologiques à l'intérieur desquels ils se mouvaient, identifier les contextes politiques au sein desquels ils manœuvraient par leur conduite comme par cette autre forme d'action qu'est l'écriture politiquement orientée.

Dans sa vaste étude sur les fondements de la pensée politique moderne, Skinner retrace minutieusement les développements qui ont mené à la cristallisation du vocabulaire politique qui nous est le plus familier: la conception moderne de l'État, les notions de souveraineté, de droits, de liberté, d'obligation ${ }^{14}$. Il montre la dépendance étroite de cette évolution envers un contexte pratique largement dominé par les activités guerrières ${ }^{15}$. Pour nous qui sommes encore situés sur le plan de l'intersubjectivité dans le monde survolé par Skinner, qui avons à nous débattre dans les environnements idéologiques conventionnels vieux de plusieurs siècles, l'approche historique peut s'avérer salutaire. Elle démystifie la nature de la pensée et de l'action politiques, faisant prendre davantage conscience aux acteurs politiques d'aujourd'hui de tout ce qui les rattache au passé. Une telle «historicisation" ne conduit pas à l'impuissance. En permettant aux penseurs et aux acteurs de mieux cerner les frontières du possible, elle mène à des délibérations politiques davantage susceptibles de respecter les exigences aristotéliciennes de la raison pratique.

Il nous semble y avoir un parallèle étroit entre l'approche historique ci-haut élaborée et les diverses tâches imputées à la

14. Skinner, p. X.

15. Pour une analyse des travaux de Skinner allant dans cette direction, voir James Tully, "The Pen is a Mighty Sword: Quentin Skinner's Analysis of Politics", British Journal of Political Science, XIII, 1983, pp. 505-506. 
théorie politique par Dunn. Pour présenter d'une façon cohérente, convaincante, les éléments constitutifs d'alternatives possibles à notre situation politique actuelle, il faut savoir se déplacer à l'intérieur de conventions idéologiques intersubjectivement acceptées; pour faire cela intelligemment, pour suggérer des modifications aux dites conventions, il faut une certaine familiarité avec les circonstances historiques de leur apparition et de leur enracinement. Avant de passer aux alternatives, Dunn insistait sur la nécessité d'une analyse et d'une évaluation des divers aspects de notre situation éthico-politique. Encore une fois, il appert impensable de procéder sur ces sentiers sans recourir à l'histoire.

Il est intéressant de noter que le thème de l'interdépendance entre théorie politique et histoire représente le terrain d'un rapprochement entre Dunn et C.B. Macpherson, habitués plutôt à ferrailler à propos de l'interprétation de Locke $^{16}$. S'il faut se pencher sur les modèles successifs de la démocratie libérale dans l'histoire, selon Macpherson, c'est parce que cela «réduit le risque de myopie lorsqu'on envisage l'avenir ${ }^{17}$. L'histoire rompt l'envoûtement qui nous colle littéralement aux paramètres de la réalité, elle nous rend disponibles en quelque sorte pour un avenir ouvert. Également, Macpherson croit que la démarche historique permet une meilleure compréhension du système présidant à notre actualité politique.

Nous nous servirons dans la suite de ce texte de l'approche historique pour élargir la réflexion sur les théories de la liberté et de la démocratie. Que le libéralisme n'échappe pas à l'attraction de l'histoire, telle est la conclusion d'un de ses plus récents analystes:

«Le libéralisme devrait être considéré, non pas en des termes fixes et abstraits, comme un assemblage de valeurs politiques et morales immuables, mais plutôt

16. À propos des divergences entre Dunn et Macpherson, dans un texte assez pessimiste sur le libéralisme, voir John Dunn, Western Political Theory in the Face of the Future, Cambridge: Cambridge University Press, 1979, p. 37.

17. C.B. Macpherson, Principes et limites de la démocratie libérale, Montréal/Paris: Boréal Express/ La Découverte, 1985, p. 9. 
comme un mouvement historique d'idées spécifique à l'ère moderne qui commence avec la Renaissance et la Réforme. En tant que tel il a connu plusieurs transformations, et requiert une forme historique d'analyse plutôt qu'une approche purement conceptuelle et statique de par sa nature ${ }^{18}$.

\section{Liberté et individualisme atomiste}

Il y aurait une vision «libertaire» de la liberté, valorisant l'épanouissement optimal des gens, l'accomplissement intégral de leurs potentialités ${ }^{19}$. Des libéraux, comme Dion, notent la difficulté de définir ce qu'est une personne vraiment épanouie, celle de préciser le comment d'un tel parachèvement des potentialités. Pareilles incertitudes paveront la voie à l'alternative qu'ils préféreront, celle de la liberté «libérale»: «la défense du quant-àsoi, de la sphère d'initiative et d'autonomie individuelle» ${ }^{20}$. Une telle vision appartient à une véritable philosophie de l'individualisme. On y valorise le libéralisme parce qu'il véhicule la conception d'un État limité, laissant suffisamment d'espace aux individus pour qu'ils puissent assumer la responsabilité de leurs choix et de leurs actions. Si le principe de l'intervention de l'État est reconnu, ce sera pour protéger la liberté des individus ${ }^{21}$. L'idéal du libéralisme, c'est donc celui de "l'individu indépendant, capable d'assumer librement la responsabilité de ses décisions» 22 .

De telles reformulations rejoignent les philosophes de l'individualisme atomiste aux dix-septième siècle, Hobbes et Locke. Elles succombent comme ces derniers penseurs aux charmes de

18. Anthony Arblaster, The Rise and Decline of Western Liberalism, Oxford: Basil Blackwell, 1984, pp. 11-12.

19. Dion, p. 16.

20. Ibid., p. 15.

21. C'est ce qu'affirment aussi les libéraux d'une autre génération. Pour une formulation récente, voir Léon Dion, $A$ la recherche du Québec, Québec: Presses de l'Université Laval, 1987, p. 13.

22. Dion, «Libéralisme et démocratie: plaidoyer pour l'idéologie dominante", p. 21. 
l'atomisme politique: une pensée qui place la création de conditions pour la réalisation de buts individuels au cœur des motifs pour l'édification de la société civile, qui affirme aussi la priorité de l'individu et de ses droits contre la société ${ }^{23}$. Une telle priorité impliquera logiquement que l'on fasse passer la liberté «libérale» avant l'égalité au mérite (l'égalité des chances) ${ }^{24}$. Selon Taylor, l'individualisme atomiste est pratiquement inégalé dans le monde contemporain sur le plan des virtualités persuasives. Il peut s'appuyer sur la très large acceptation de l'identité moderne, celle d'un sujet désincarné. La révolution scientifique et la révolution philosophique dans la mouvance cartésienne ont contribué à créer une identité pour l'être humain caractéristique de la modernité. L'être humain ne se définit plus désormais par la place qu'il occupe dans la grande chaîne aristotélicienne de l'être; sa nouvelle indépendance est annonciatrice de transformations radicales de son mode de vie. Dans la modernité, l'être humain pourra contrôler la nature, l'objectiver, la soumettre à ses propres fins. Grâce à la science, il s'est arraché aux contraintes de la nature, de nouvelles avenues se sont ouvertes à lui, ou à elle, sur le plan de l'action. Le nouvel espace neutre qu'il occupe en face de la nature, la nouvelle liberté qui est sienne à son égard, l'être humain voudra les transplanter dans l'ordre de la société, de l'économie et de la politique. Mieux à même de juguler les interventions de la nature dans sa vie, l'être humain se repliera sur lui-même pour associer liberté et non-interventionnisme de la part d'autrui ${ }^{25}$.

Personne parmi les libéraux atomistes n'échappe à la nécessité de concevoir philosophiquement et moralement la nature humaine.

23. Charles Taylor, "Atomism", dans Charles Taylor, Philosophy and the Human Sciences, (Philosophical Papers. vol. II), Cambridge: Cambridge University Press, 1985, p. 187. p. 15 .

24. Dion, "Libéralisme et démocratie: plaidoyer pour l'idéologie dominante",

25. Taylor, "Introduction», dans Taylor, Philosophy and the Human Sizences. (Philosophical Papers, vol.II), p. 5. 
Cet être que l'on veut protéger dans toute la sphère de sa liberté, il faut bien lui attribuer une valeur particulière, reconnaître sa rationalité distinctive et sa capacité de faire des choix. On doit remarquer que ce que les libéraux atomistes eux-mêmes valorisent dans l'existence humaine ne se présente pas comme un donné pur et simple. Agir rationellement, faire des choix lucides, ce sont des qualités humaines qui apparaissent d'abord au rang de potentialités, et qui requièrent par après un lourd bagage social pour pouvoir espérer s'actualiser. Logiquement, nous semble-til, la société est en droit de s'attendre à un certain nombre de devoirs, d'obligations, dont celle d'appartenance, de la part des individus qui ont besoin d'elle pour réaliser leurs virtualités ${ }^{26}$.

Il est possible, dans la tradition libérale, de refuser le principe de ces obligations en poussant l'atomisme jusqu'aux confins de l'a-socialité. Chez Hobbes, père en ce sens de l'individualisme atomiste, les humains sont des êtres de désir, assoiffés de pouvoir de façon à désirer et à obtenir davantage, privilégiant par-dessus toute autre chose le maintien de leur mouvement vital ${ }^{27}$. Excluant toute référence à la notion de formes supérieures de vie, la pensée ici reste rivée aux objectifs de sécurité, de préservation. Pour assurer son triomphe, le libéralisme a-social doit toutefois payer le prix fort. Il est obligé de se contenter d'un nombre réduit de droits individuels: droit à la vie, à la satisfaction des désirs, à la liberté de désirer. Or l'autonomie humaine est irréductible à la liberté de désirer:

«... la liberté de choisir des projets de vie, de disposer de ses possessions, d'en arriver à ses propres convictions et d'agir raisonnablement à partir d'elles, etc... Mais alors on a affaire à des aptitudes qui ne nous appartiennent pas du simple fait que nous soyons vivants - des aptitudes qui à tout le moins en certaines

26. Taylor, "Atomism», p. 198.

27. Voir la conception de l'homme qui est présentée dans Thomas Hobbes, Leviathan. New-York: Macmillan Publishing Company, 1962, chapitre 6, pp. 47ss. 
occasions pourraient ne pas être adéquatement développées; c'est ainsi que se pose la question des conditions propices à leur développement ${ }^{28}$.

Faire des choix, assumer ses responsabilités, mener une existence autonome, être capable de donner un sens à sa vie, voilà autant de qualités, de possibilités que même les libéraux atomistes accordent à la vie humaine, et voilà autant de réalités qui ne peuvent voir le jour que dans un contexte civilisationnel très avancé. Les atomistes contemporains, à leur corps défendant, ne réussissent pas à éviter la conception de la liberté en tant qu'épanouissement, qu'auto-réalisation de l'individu. Ils ne peuvent se satisfaire ultimement du modèle dur de la liberté dite négative, l'absence d'obstacles, d'empêchements externes au comportement de l'individu ${ }^{29}$. Un individu libre, pour eux, c'est davantage qu'un ensemble hobbésien de particules capable de poursuivre son chemin sans interférence; c'est aussi, et peut-être même surtout, un être apte à la délibération rationnelle, à l'agir responsable, tout en étant source première d'attribution de sens pour sa propre $\mathrm{vie}^{30}$.

L'auto-compréhension moderne, faite d'une forte valorisation de l'autonomie individuelle, nous la devons aux conditions historiques d'évolution de notre civilisation. Il s'agit donc d'une identité située, contingente, dépendante par rapport à une série de développements dans une pluralité de domaines tels la science et la technologie, les arts et la politique, la philosophie et

28. Taylor, "Atomism», p. 202.

29. Isaiah Berlin, Four Essays on Liberty, London: Oxford University Press, 1969, pp. 122ss.

30. Dion trouvera peut-être quelque consolation à l'idée que John Stuart Mill, qu'il place à juste titre parmi les figures marquantes du libéralisme, était lui aussi écartelé entre la liberté "absence d'empêchements", et la liberté «épanouissement de l'individualité». Voir John Stuart Mill, On Liberty, Harmondsworth: Penquin Books, 1980, pp. 67ss. pour la liberté «absence d'empêchement», p. 121 pour la liberté «réalisation de l'individualité». 
l'économie $^{31}$. Ces développements ont produit des institutions qui sont les véritables remparts de la liberté et de l'autonomie de l'individu. En de telles circonstances, l'individu est convié à se soucier du sort de telles institutions dans la société politique à laquelle il appartient. Nous arrivons ici à un point où les limites de la liberté à l'intérieur de l'individualisme atomiste se doublent de celles de la démocratie de représentation.

\section{Démocratie antique et humanisme républicain}

La démocratie directe et les idéaux de la participation n'ont pas très bonne presse auprès des libéraux des années quatre-vingt ${ }^{32}$. Ces derniers tiennent pour particulièrement évidents et progressistes les postulats ontologiques et métaphysiques transformant le sujet moderne en une forteresse quasi-autarcique. Parfaitement à l'aise comme nous l'avons constaté dans le cadre de la pensée atomiste, ils déduisent à partir de semblables postultats, à la façon de Benjamin Constant, une vision exclusivement moderne de la démocratie et surtout de la liberté, fondées sur les droits individuels comme celui de propriété et sur la prépondérance de l'existence privée. Logiquement, un libéral comme Dion n'a pas nécessairement tort lorsqu'il affirme que la démocratie directe n'a jamais existé. Si l'on entérine comme définition de ce phénomène l'idée d'un gouvernement du peuple par le peuple, où le peuple exprimerait sa souveraineté par une espèce d'ubiquité qui le ferait tout à la fois délibérer, décider, légiférer, exécuter, administrer voire juger, il est bien clair qu'en ce sens optimal la démocratie directe s'avère chimère. Il faut d'ailleurs se remémorer le réalisme de Rousseau,

31. Taylor, "Atomism», p. 204.

32. Dion, "Libéralisme et démocratie: plaidoyer pour l'idéologie dominante", pp. 8-9. 
qui allait jusqu'à l'affirmation de l'impossibilité de la démocratie vraie, idéale ${ }^{33}$.

Dion, toutefois, va beaucoup plus loin que la simple négation historique de la démocratie directe dans l'atmosphère raréfiée de son type idéal. Ses oppositions préférées, individualismecollectivisme, démocratie libérale-socialisme de planification, illustrent bien le fait qu'il n'y a pas de place dans sa conception de la politique pour les visions antiques de la démocratie et de la liberté. Ces dernières n'ont pas été inventées a posteriori par les historiens. La négligence de leur signification politique a été facilitée, pour notre modernité libérale, par les critiques d'Aristote et de Platon. Aristote n'est cependant pas un adversaire absolu de la démocratie antique ${ }^{34}$. On peut trouver dans La politique des réflexions qui sont les compagnes philosophiques des études historiques sympathiques à la démocratie antique. Sur le plan de la raison pratique le peuple rassemblé n'aurait rien à envier à personne. Sa sagesse, sa capacité de juger lorsqu'il est constitué en corps de délibération, seraient insurpassées. ${ }^{35}$.

Aristote fait beaucoup plus que conforter nos préjugés démocratiques modernes. Il nous sensibilise à une tout autre vision de la politique. La participation du citoyen est bel et bien chez lui une nécessité pour la "polis»; sauf que cela ne se confine pas à l'apprentissage de la démocratie, à l'obtention des prérequis qui assureront une qualité de vie démocratique ${ }^{36}$. Les citoyens ne peuvent se réaliser éthiquement, ils ne peuvent prétendre goûter

33. Jean-Jacques Rousseau, Du contrat social, Paris: Garnier-Flammarion, 1966, Livre III, chapitre IV, p. 107.

34. Le régime qu'Aristote favorise, le régime mixte ou "politeia", contient d'importants éléments démocratiques. Voir Aristote, The Politics, London: Oxford University Press, 1958, Livre IV, chapitre 8, pp. 174-176.

35. Ibid., Livre III, chapitres X et XI, pp. 121-127.

36. Dion, «Libéralisme et démocratie: plaidoyer pour l'idéologie dominante», p. 10 . 
à "l'eudaimonia», à la félicité, que s'ils sont actifs à l'intérieur de cette communauté de délibération qu'est la polis. Nous voici bien loin des accents utilitaristes avec lesquels Dion évalue la participation dans les sociétés modernes, réduite à «un coût que la plupart des citoyens préferent affecter à d'autres fins en l'absence de menaces immédiates contre leurs intérêts ${ }^{37}$.

La philosophie d'Aristote mène donc à l'identification des piliers de cette tradition de pensée et de pratique politiques mise à l'écart dans les catégories de l'individualisme libéral. Cette tradition, c'est celle de l'humanisme civique, ou de l'humanisme républicain. Hannah Arendt a contribué à récupérer pour notre temps toute la force de cette notion de l'agir commun dans la vie politique athénienne ${ }^{38}$. Le sommet de la condition humaine pour elle se trouve dans les espaces publics où les humains dévoilent en quelque sorte leur être dans l'action et dans la communication. S'il y a bel et bien eu une invention de la politique, cela fut le fait de ces citoyens anonymes qui quittaient fréquemment leurs champs pour venir apporter leur contribution aux délibérations de «l'ecclesia» ${ }^{39}$. On admettra volontiers que le degré de participation variait selon l'importance des thèmes discutés, atteignant un degré maximal lorsqu'il fallait décider du principe d'une entreprise guerrière. Cette nuance n'emporte pas l'invalidité de la distinction qualitative entre cette politique à l'ancienne, toute axée sur la délibération et l'agir communs, sur la prise en charge des magistratures à tour de rôle par les citoyens, et la politique libérale moderne à l'intérieur de la démocratie de représentation. Pour Hannah Arendt encore une fois «l'hubris», la fierté de

37. Ibid., p. 22.

38. Hannah Arendt, The Human Condition, Chicago: University of Chicago Press, 1958, pp. 24-25, pp. 197-198. Voir aussi Jürgen Habermas, Theory and Practice, Boston: Beacon Press, 1973, p. 42.

39. Moses Finley, L'invention de la politique, Paris: Flammarion, 1985, pp. 105110. 
Périclès dans les discours que lui attribue Thucydide est parfaitement

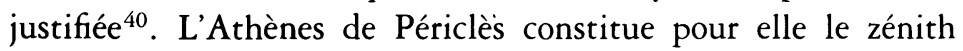
parmi les communautés politiques de l'histoire de l'Occident.

Lorsqu'on articule une réflexion théorique à l'abri de l'histoire, on peut se permettre d'écarter assez rapidement ceux qui ont la nostalgie de la démocratie directe. Après tout, pourrait-on nous rétorquer, les partisans de l'humanisme civique ont beau s'épancher sur la dégénérescence de la politique à l'ère moderne, il n'en demeure pas moins que leurs exemples privilégiés, Athènes et la Rome républicaines, sont complètement déconnectés des réalités complexes de la politique d'aujourd'hui. Pour répondre à cela, nous nous pencherons maintenant sur certaines des manifestations historiques qui servent de médiatrices entre la pratique de l'Antiquité et les formulations actuelles dans le discours de l'humanisme civique.

C'est en Italie, du treizième au seizième siècles, que l'on vit réapparaître dans la sphère idéologique et dans la pratique politique, le vieux concept romain de «libertas». Ce concept se présentait sous deux couches sémantiques: d'abord l'autonomie par rapport aux autres unités politiques, ensuite et surtout pour notre propos la participation des citoyens à la direction des affaires publiques $^{41}$. Pour cet humanisme républicain qui atteignit les plus hauts sommets durant le quattrocento florentin, les citoyens pouvaient prétendre à la vertu lorsqu'ils faisaient fructifier leurs talents et déployaient leurs énergies en se mettant au service de la communautée ${ }^{42}$. Le Prince de Machiavel est bien loin de constituer la seule grande contribution de ce penseur à la théorie politique. Ses Discours nous fournissent un des plus beaux exemples de la défense de l'esprit républicain dans l'Italie de la Renaissance. La

40. Thucydide, Histoire de la guerre du Péloponnèse. Paris: Garnier-Flammarion, 1966, Livre I, chapitres 140ss, Livre II, chapitres 60ss.

41. Skinner, vol. I, p. 54.

42. Ibid., p. 80 . 
liberté d'une cité, sa sauvegarde contre les servitudes internes autant qu'externes, reposaient ultimement sur la «virtu» des citoyens prenant le contrôle du gouvernement, sur la vitalité des différents camps en conflit les uns avec les autres pour le plus grand bien de l'ensemble ${ }^{43}$.

Le début du dix-septième siècle marque une période de retraite pour l'humanisme républicain. Presque complètement évincé de la politique italienne, il subit aussi des revers idéologiques importants dans un pays comme la France. L'absolutisme politique y fut accompagné par un renouvellement de la critique augustinienne du républicanisme ${ }^{44}$. C'est dans une Angleterre prise dans les tourments de la guerre civile que la pensée républicaine retrouva toute sa vigueur au dix-septième siècle, particulièrement dans le livre de James Harrington, Oceana ${ }^{45}$. Bien qu'il dût s'avérer vaincu en Angleterre, le républicanisme ne perdit pas tout. La culture politique des colonies américaines, au dix-huitième siècle, était fortement imprégnée des valeurs de l'humanisme civique néoharringtonien ${ }^{46}$. L'influence de Montesquieu sur la politique américaine du dix-huitième siècle ne fut pas restreinte à la théorie de la séparation des pouvoirs. Montesquieu rejoignait l'humanisme républicain lorsqu'il écrivait dans L'Esprit des lois que la liberté n'était pas l'indépendance, pas plus qu'elle n'était selon le langage

43. Nicolo Machiavelli, The Discourses. Harmondsworth: Penquin Books, 1974, pp. 113-114 pour l'appréciation des conflits et tumultes publics par l'auteur, pp. 252 254 , pour ses commentaires positifs quant aux potentialités des masses populaires dans l'action politique.

44. Nannerl Keohane, Philosophy and the State in France. Princeton: Princeton University Press, 1980 , pp. 83ss.

45. J.G.A. Pocock, The Machiavellian Moment, Princeton: Princeton University Press, 1975, pp. 384-385.

46. Ibid., pp. 506-507. Dion inclut Jefferson dans son énumération des grands penseurs de la tradition libérale. C'est toutefois bien plus de Madison que de Jefferson qu'il devrait se réclamer dans sa conception de la démocratie libérale axée sur la représentation. Jefferson, lui, alliait humanisme civique et affirmation des mérites de la propriété terrienne. Voir à ce sujet J.G.A. Pocock, Politics, Language and Time, New-York: Atheneum, 1971 , pp. 98-99. 
libéral contemporain la défense du quant-à-soi. Elle consistait plutôt à pouvoir faire ce que l'on devait faire, à mener une vie en sûreté sous l'égide des lois ${ }^{47}$. Les Américains pouvaient aussi retrouver chez Montesquieu de belles phrases sur le rôle de la vertu dans l'état politique, sur l'amour de la république dans les démocraties, sur ce que certains de nos contemporains appellent un sain patriotisme ${ }^{48}$.

L'esprit de l'humanisme républicain, c'est bien entendu aussi celui de Rousseau. Il ne peut être de notre propos ici de discourir sur le bien-fondé des accusations de précurseur du totalitarisme qui ont été lancées à Rousseau dans l'après-guerre. Il est certain qu'en parlant de forcer les gens à être libres, Rousseau a contraint maints penseurs à renoncer à l'idée d'une liberté positive, entendue au sens du contrôle collectif sur la vie commune, et à retraiter vers une approche négative ${ }^{49}$. Sur le plan de l'histoire des idées, toutefois, il faut constater que la puissance expressive de Rousseau dans son attribution de la souveraineté dans la cité à la volonté générale, dans son identification de la liberté avec l'obéissance aux lois votées par le corps souverain, a eu une influence déterminante en France ${ }^{50}$. Au fil des décennies, la critique de Constant n'a pas réussi à oblitérer la marque du républicanisme de Rousseau sur les institutions et l'esprit politique français. En France, il n'y aurait pas eu de césure radicale entre la liberté des Anciens et la liberté des Modernes. ${ }^{51}$.

Les penseurs de notre époque qui poursuivent la tradition de l'humanisme républicain, tels Arendt, Habermas et Taylor, sont bien loin de se complaire uniquement dans une nostalgie

47. Montesquieu, De L'esprit des lois, Paris: Éditions Garnier, 1973, tome I, Livre XI, chapitre III, et Livre XII, chapitre II. p. 110 .

48. Ibid., livre V, chapitres 2 et 3. Voir aussi Dion, À la recherche du Québec,

49. Berlin, p. 161.

50. Rousseau, Livre II, chapitres 1 et 2.

51. Claude Nicolet, L'idée républicaine en France, Paris: Gallimard, 1982, p. 480. 
passéiste de l'âge d'or athénien. Ils peuvent trouver des références pratiquement à toutes les époques, dans toutes les branches de la civilisation occidentale. Ce qu'ils recherchent pour une époque troublée comme la nôtre, c'est ce que Hegel appelait une «Sittlichkeit», une moralité publique objective. Une telle moralité accrédite l'existence d'obligations liant les êtres humains à une vie qui les englobe, qu'ils doivent supporter de leurs efforts ${ }^{52}$. Dans une société où existe une «Sittlichkeit», il y a un bien qui n'est pas uniquement le mien, mais celui de la communauté.

S'il y a une carence fondamentale dans le libéralisme atomiste moderne, c'est bien son refus d'accorder toute autonomie, même partielle, à la notion de bien commun. Ce dernier ne peut signifier pour le libéralisme qu'un agrégat d'intérêts individuels ${ }^{53}$. La pensée libérale, lorsqu'elle est généreuse, croit trouver en son sein les armes idéologiques qui lui permettront de justifier l'intervention de l'État pour rétablir l'égalité des chances entre les individus. Que le libéralisme puisse contenir d'autre part les outils requis pour lutter contre les tenants de l'État minimal, nous ne le nierons pas. Cela ne signifie toutefois pas qu'il possède les ressources discursives pour faire face à la réalité politique contemporaine. L'individualisme atomiste, les calculs utilitaristes qui l'accompagnent, sont incompatibles avec l'idée d'une obligation pour les êtres humains d'agir en vue de la défense des pratiques et des institutions constitutives d'une civilisation. Pourtant, comme nous l'avons rappelé avec Taylor dans la section précédente, c'est cet héritage civilisationnel pluri-séculaire qui rend possible l'émergence d'individus autonomes, responsables, capables de faire des choix

52. Charles Taylor, Hegel and Modern Society, Cambridge: Cambridge University Press, 1979, p. 125.

53. Dion, "Libéralisme et démocratie: plaidoyer pour l'idéologie dominante, pp. 22 et 27 . On cherchera en vain dans ce texte toute référence à l'idée de bien commun. Il y est bien question de communauté en quelques occasions, mais cela se produit dans des passages où la mention de ce mot prend des connotations péjoratives. 
lucides pour orienter leur vie ${ }^{54}$. On est en droit de se demander comment les êtres humains pourraient accepter de faire les sacrifices requis par la lutte pour la sauvegarde de semblables piliers civilisationnels, pour la protection de l'environnement, voire même dans le contexte québécois pour le maintien de l'intégrité et de la spécificité d'un groupe culturel et linguistique, sans l'atout d'une vision plus généreuse du bien commun que ne l'autorise la pensée libérale dans certaines de ses formulations les plus actuelles.

La récession des années quatre-vingt a provoqué un durcissement des régimes politiques en Occident, un retour en force du conservatisme comme une contraction de la pensée libérale, un repli vers ce noyau dur qu'est pour elle l'individualisme atomiste. La stabilité actuelle ne doit pas faire illusion ${ }^{55}$. Ces régimes n'ont pas résolu les problèmes qui les prenaient à la gorge il y a une quinzaine d'années:

«... incapacité de procurer aux individus et aux collectivités le sens de la dignité qu'ils réclament et qu'ils acquerraient si on leur permettait de gagner leur vie par leur travail et de participer activement au contrôle de leur environnement; impuissance à formuler des objectifs collectifs propres à susciter un vaste courant de confiance et de loyauté envers les grandes organisations de gestion, les Églises, les gouvernements, les institutions d'enseignement, les entreprises économiques, les professions, les unions de travailleurs; ... ${ }^{56}$

Pour accueillir ouvertement une vision du bien commun, la pensée libérale devrait reconsidérer ce qu'elle entend par communauté et par politique. Une brève analyse de ces deux notions mettra un terme à notre survol de la tradition républicaine. En simplifiant, on peut ramener la pensée libérale à une théorie instrumentale de la communauté, trouvant dans la société politique les préconditions pour la satisfaction des intérêts égoïstes, pour

54. Taylor, "Atomism», pp. 207-208.

55. Léon Dion émettait un jugement semblable à propos de l'accalmie du début des années soixante-dix. Voir Léon Dion, Société et politique: la vie des groupes (tome second: dynamique de la société libérale), Québec: Presses de L'Université Laval, 1972, p. 469.

56. Léon Dion, La prochaine révolution, Montréal: Leméac, 1973, p. 323. 
la quête tranquille du quant-à-soi. La définition de l'identité individuelle dans ses aspects essentiels précède selon ce modèle l'association politique. Les partisans de l'humanisme républicain militent plutôt en faveur d'une théorie constitutive de la communauté $^{57}$. Les liens rattachant l'individu à la communauté ici ne lui sont pas extrinsèques. Ils appartiennent à son identité, ils en représentent un morceau essentiel. Privé de la communauté politique en question, un tel individu ne serait qualitativement plus le même. Tout lecteur saura trouver dans sa mémoire des exemples pertinents de personnes qui ne seraient littéralement plus les mêmes, sevrées de leur communauté politique d'appartenance. Dans la tradition républicaine, cela ne vaut pas uniquement pour les personnalités d'exception, mais pour chacun d'entre nous. La loyauté qu'un individu doit à sa communauté, en vertu même de son identité, n'occupe pas le même statut sur le plan moral que les obligations qu' il contracte avec ses voisins. Dans le premier cas, un retrait équivaudrait à une transformation de l'être même de l'individu.

À l'intérieur d'une communauté entendue au sens constitutif, l'humanisme républicain attribue la plus haute valeur à l'activité politique. Cette dernière jour un rôle fondamental dans l'évolution de l'identité des citoyens. Grâce au processus de dialogue que l'on place au cœur de la politique, les citoyens sont susceptibles de gagner en lucidité personnelle ${ }^{58}$. Leurs valeurs morales et leurs objectifs s'en trouvent clarifiés, mieux définis. Une telle vie politique correspond à un enrichissement, dans le sens le plus noble du terme, pour tous les participants. La quête de l'identité individuelle est ici achevée, complétée, grâce à l'exercice par chaque citoyen

57. Michael Sandel, Liberalism and the Limits of Justice, Cambridge: Cambridge University Press, 1982, p. 150.

58. Ronald Beiner, Political Judgment, Londres: Methuen, 1983, p. 152. 
de ses aptitudes au jugement politique. Renoncer à cela, ce serait consentir à un appauvrissement de notre identité.

\section{Conclusion}

«Le libéralisme refuserait de restreindre la responsabilité pour la prudence collective à une petite élite de gouvernants, il affirmerait plutôt que la plus grande partie de cette responsabilité devrait être portée, pour qu'elle eût quelque chance d'être menée à bien, par la solidarité, la vertu civique et le discernement politique de l'ensemble des citoyens" "99.

Le libéralisme, dans l'optique de John Dunn, n'est pas incompatible avec les idéaux de l'humanisme républicain. Pour qu'une telle synthèse fût possible, il faudrait toutefois que la théorie politique renouât avec la démarche historique. Les idéaux d'autonomie individuelle et de responsabilité dans les choix et les actions seraient alors assumés dans une pensée plus ouverte sur la société, plus consciente des devoirs de l'appartenance et des bénéfices de la participation. Vu la taille des cités politiques contemporaines, il serait illusoire de renoncer à la démocratie de représentation pour installer la permanence et le monopole des délibérations de l'ensemble de la communauté ${ }^{60}$. Entre la dévalorisation de la participation et sa tout aussi imprudente déification, il devrait y avoir de la place pour des aménagements qui allieraient le souci de l'efficacité à celui d'une vie politique significative pour le plus grand nombre ${ }^{61}$.

Nous avons élaboré dans cet article un plaidoyer pour la reconnaissance de l'apport essentiel de l'histoire à la démarche de

59. John Dunn, Rethinking Modern Political Theory, Cambridge: Cambridge University Press, 1985, pp. 168-169.

60. Voir les essais de C.B. Macpherson, Principes et limites de la démocratie libérale, Montréal/Paris: Boréal Express/La Découverte, 1985, et de Philip Resnick, Parliament vs. People: An Essay on Democracty and Canadian Political Culture, Vancouver: New Star Books, 1984.

61. Voir Dion, Société et politique: la vie des groupes (tome second: dynamique de la société libérale), p. 476. Également Dion, La prochaine révolution, pp. 194 et 262, pour un refus de la dévalorisation de la participation. 
la théorie politique. L'histoire nous a servi de clé pour identifier quelques apories dans la réflexion articulée par Stéphane Dion autour des idées de libéralisme et de démocratie. L'individualisme atomiste de Dion, son omission de tout un pan de la pensée politique occidentale, à savoir l'humanisme républicain, nous semblent représentatifs de la pensée libérale contemporaine. En puisant à même la tradition républicaine, nous avons donné quelques indications de ce que pourrait être une conception élargie de la liberté, de la démocratie. L'histoire des idées politiques, encore elle, nous permet de constater que les temps ont bien changé au Québec.

«Il est des contraintes, et elles sont nombreuses et de tous genres, que les citoyens doivent savoir s'imposer pour le bien général " ${ }^{62}$.

Il n'y a pas si longtemps, les partisans les plus en vue du libéralisme n'avaient pas encore rompu les ponts avec la tradition républicaine, avec une sympathie bien sentie pour l'idée de bien commun. Résolument modernes, fiers d'adhérer à une pensée qui avait reconnu la primauté de l'individu et de ses droits, ils n'avaient aucune envie de renouer avec les théories corporatistes et organicistes de l'État qui avaient cours naguère chez nous ${ }^{63}$. Ils avaient toutefois la sagesse de reconnaître que le libéralisme n'épuise pas la tradition humaniste occidentale ${ }^{64}$. Ils s'inspiraient, et s'inspirent encore, de cette dernière pour enrichir leur libéralisme du vocabulaire des Anciens: patriotisme, vertus civiques, devoirs moraux envers la communauté politique d'appartenance ${ }^{65}$. Confrontés aux choix politiques de leur génération, les libéraux d'aujourd'hui finiront bien, c'est le pari que nous faisons, par retrouver le chemin de la tradition républicaine.

62. Dion, La prochaine révolution, p. 88.

63. Ibid., p. 334. Voir aussi Léon Dion, Le Bill 60 et la société québecoise, Montréal : H.M.H., 1967, p. 153.

64. Dion, La prochaine révolution, p. 276.

65. Dion, À la recherche du Québec, pp. 110 et 164. 